\title{
A comprehensive characterization of the caspase gene family in insects from the order Lepidoptera
}

\author{
Juliette Courtiade, Yannick Pauchet", Heiko Vogel and David G Heckel
}

\begin{abstract}
Background: The cell suicide pathway of apoptosis is a necessary event in the life of multicellular organisms. It is involved in many biological processes ranging from development to the immune response. Evolutionarily conserved proteases, called caspases, play a central role in regulating apoptosis. Reception of death stimuli triggers the activation of initiator caspases, which in turn activate the effector caspases. In Lepidoptera, apoptosis is crucial in processes such as metamorphosis or defending against baculovirus infection. The discovery of p35, a baculovirus protein inhibiting caspase activity, has led to the characterization of the first lepidopteran caspase, Sf-Caspase-1. Studies on Sf-Caspase-1 mode of activation suggested that apoptosis in Lepidoptera requires a cascade of caspase activation, as demonstrated in many other species.

Results: In order to get insights into this gene family in Lepidoptera, we performed an extensive survey of lepidopteran-derived EST datasets. We identified 66 sequences distributed among 27 species encoding putative caspases. Phylogenetic analyses showed that Lepidoptera possess at least 5 caspases, for which we propose a unified nomenclature. According to homology to their Drosophila counterparts and their primary structure, we determined that Lep-Caspase-1, -2 and -3 are putative effector caspases, whereas Lep-Caspase-5 and -6 are putative initiators. The likely function of Lep-Caspase-4 remains unclear. Lep-Caspase-2 is absent from the silkworm genome and appears to be noctuid-specific, and to have arisen from a tandem duplication of the Caspase-1 gene. In the tobacco hawkmoth, 3 distinct transcripts encoding putative Caspase-4 were identified, suggesting at least 2 duplication events in this species.
\end{abstract}

Conclusions: The basic repertoire of five major types of caspases shared among Lepidoptera seems to be smaller than for most other groups studied to date, but gene duplication still plays a role in lineage-specific increases in diversity, just as in Diptera and mammals.

\section{Background}

Apoptosis, a distinctive and highly regulated type of cell suicide, is fundamental for various biological processes such as development [1], tissue homeostasis, DNA damage response [2] and immune response [3]. Development of holometabolous insects is characterized by a complete metamorphosis between the wingless larval stage, mostly dedicated to nutrient acquisition and growth, and the winged adult form, dedicated to reproduction. These drastic modifications in appearance and physiology require massive histolysis and histogenesis. The importance of apoptotic events during development of holometabolous insects and more specifically in Lepidoptera has been

\footnotetext{
* Correspondence: ypauchet@ice.mpg.de

Department of Entomology, Max Planck Institute for Chemical Ecology, Hans-Knöll-Str. 8, 07745 Jena, Germany
}

shown as early as the 1960 's. In wild silkmoths and the tobacco hawkmoth, the first ecdysone peak during metamorphosis induces apoptotic degeneration of the larval intersegmental muscles, proleg motoneurons, and labial glands [4-6]. The decrease in ecdysone titer shortly before hatching induces apoptotic degeneration of abdominal neurons and intersegmental muscles [7]. Important changes in food habits between larval and adult stages imply extensive remodeling of the digestive tract. For example, in Galleria mellonella, the larval midgut undergoes apoptosis during metamorphosis [8]. Similarly, in Heliothis virescens, apoptosis of the larval midgut has been correlated with higher caspase expression shortly before and after pupation [9]. Among the different protective measures triggered by the insect immune system to thwart pathogen infection, host cell suicide through apoptosis can 
significantly reduce viral replication, dissemination and infectivity [10].

A major family of evolutionarily conserved cysteinedependent aspartate-specific proteases, called caspases, plays a central role in apoptosis. Studies on alpha-proteobacteria, closely related species of the free-living ancestor of mitochondria, revealed the presence of genes similar to caspases, suggesting that ancestor of eukaryotic caspases derived from mitochondrial endosymbionts [11]. Ancestors of caspase genes then evolved into 'metacaspases' in plants and fungi, 'paracaspases' in slime, molds and animals and in 'true caspases' in animals. The number of "true" caspase genes differs greatly among animal lineages with as little as 4 genes in Caenorhabditis elegans [12], 5 in the sea anemone Nematostella vectensis, 7 in the fruit fly Drosophila melanogaster [13], 10 and 11 in the mosquitoes Anopheles gambiae and Aedes aegypti respectively [14], 12 in human [15,16], to up to 31 in the sea urchin Strongylocentrotus purpuratus [17]. It has been hypothesized that the host-pathogen interaction has been one of the major evolutionary forces shaping the apoptotic machinery and therefore the caspase repertoire $[17,18]$.

The caspase family is divided into two groups: the inflammatory caspases and the apoptotic caspases composed of the initiator and the effector subgroups. To date, dedicated inflammatory caspases seem to be restricted to mammals. Although a non-apoptotic role of some lepidopteran caspases should not be excluded at this stage, the description of inflammatory caspases will not be considered further here. Apoptotic caspases are synthesized in the cell as catalytically inactive forms. All members of the caspase family share a canonical structure consisting of a prodomain and a catalytic domain named "peptidase C14" (Caspase domain pfam PF00656). The catalytic domain is formed by 2 subunits, a large one of about $20 \mathrm{kDa}$, also known as p20, and a small one of about $10 \mathrm{kDa}$, also referred as p10. Upon proteolytic processing of the proenzyme, the two subunits form a heterodimer and the active caspase enzyme is made of the association of two of these heterodimers. The enzymatic activity of caspases shows strong cleavage site specificity towards a four amino-acid motif ending with an aspartate residue [19-21]. In contrast to the catalytic domain, which is structurally conserved, the prodomain varies significantly among caspases. The characteristic binding sites $(\mathrm{L} / \mathrm{s} / \mathrm{s} H G)$ and active sites $\left(\mathrm{Q}^{\mathrm{A}} / \mathrm{R}_{\mathrm{R}} \mathrm{C}^{\mathrm{R}} / \mathrm{Q} \mathrm{G}\right)$ are conserved in all caspases except in D. melanogaster Dronc [22]. Whereas effector caspases have a short prodomain of about 30 amino acids, initiator caspases have a prodomain of 80 or more amino acids [23]. Long prodomains usually harbor structures belonging to the Death Domain superfamily, such as Death Effector Domain (DED) or Caspase Recruitment Domain (CARD), which are involved in the recruitment and subsequent activation of initiator caspases by death receptors or by the apoptosome [24].

In the pro-apoptotic proteolytic cascade, initiator caspases are activated first, and these in turn activate the effector caspases. The latter are responsible for the cleavage of many cellular components [25], leading to the characteristic features of apoptotic cells such as DNA fragmentation and membrane blebbing [26]. In mammals, in the extrinsic pathway, the binding of a "death" ligand to its receptor changes the receptor conformation, allowing the recruitment of an adaptor protein such as FADD and of initiator Caspase-8. The formation of this complex leads to the autoactivation of Caspase-8 (Figure 1). The intrinsic pathway is activated in response to intracellular signals which induce a permeabilization of the mitochondria outer membrane, allowing the release in the cytosol of several proteins such as Smac/Diablo and Cytochrome c. Cytochrome c forms a protein complex with Apaf 1 called the apoptosome, which recruits the initiator Caspase- 9 leading to its subsequent activation. Caspase- 8 and -9 are both able to activate the effector caspase-3, -6 and -7 (Figure 1). In Drosophila, there is no evidence of an extrinsic pathway, and the formation of the apoptosome with Dark, the homolog of Apaf 1, and Dronc, the homolog of Caspase-9, does not require Cytochrome c [27]. Dronc then activates the effector caspase DrICE. Homologs of the core components of the apoptosis machinery found in Drosophila have also been described in mosquitoes, suggesting that apoptosis is regulated in a similar fashion in mosquitoes [14].

In Lepidoptera, very little is known about apoptotic molecular pathways (Figure 1). The discovery in the early 1990's of p35, a baculovirus pan-caspase inhibitor [28], has led to the characterization of the first lepidopteran caspase, Sf-caspase-1, from the Sf9 cell line derived from the noctuid moth Spodoptera frugiperda [29]. Later, the likely orthologs from S. littoralis [30], Helicoverpa armigera [31] and Trichoplusia ni [32] were also characterized. Members of this caspase- 1 group have been shown to act as effector caspases, but so far no other classes of caspases have been described in Lepidoptera. The existence of an "apical" caspase responsible for the activation of Sf-caspase-1 has been suggested [33]. Furthermore, 2 other baculovirus proteins, p49 and IAP $[34,35]$ have been shown to inhibit apoptosis in Sf9 cells by blocking the processing of Sf-caspase-1, suggesting that the likely target of these 2 inhibitors may be an apical caspase, tentatively named Sf-caspase X. So far, this caspase X has not been characterized, but these results suggest that, in Lepidoptera, apoptosis requires a cascade of caspase activation, similar to other organisms.

In an attempt to characterize the caspase gene family in lepidopteran insects, we first surveyed the recently 


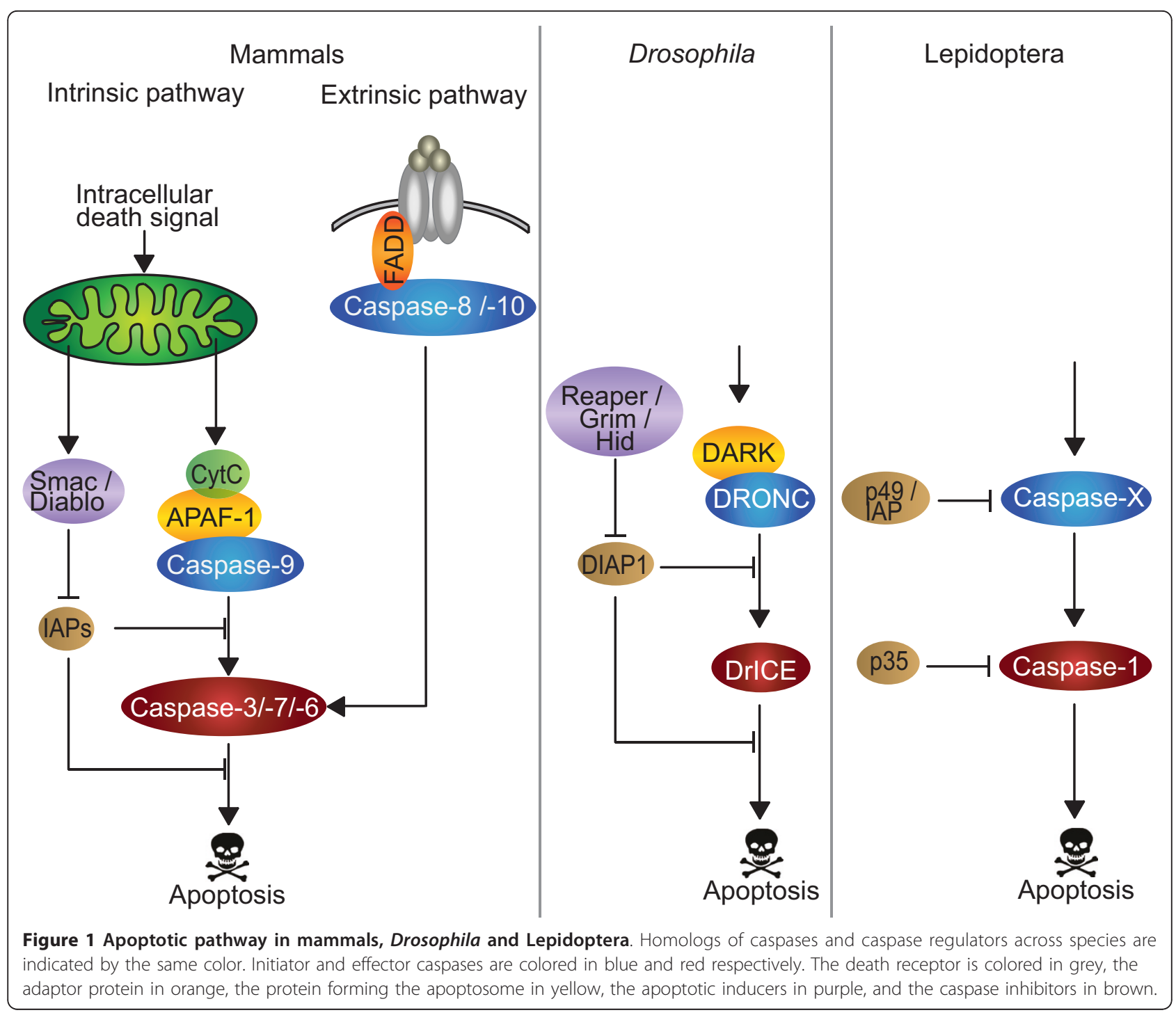

sequenced genome of the silkworm Bombyx mori [36], as well as publicly available and 'in-house' EST datasets. From these, we retrieved 66 transcripts encoding putative caspases from 27 species of butterflies and moths. Phylogenetic analyses showed that these genes clustered into 6 distinct clades (Caspase-1 to -6), three of which are classified as putative effector caspases, two corresponding to putative initiator caspases and one that could not be readily classified as either. We propose a naming convention for these genes based on our phylogenetic analyses. Finally, we discovered that one of the caspase subfamilies (caspase-2) is absent from the genome of the silkworm, has evolved from a tandem gene duplication of caspase-1, is under purifying selection which however is more relaxed than for caspase-1, and is likely to be restricted to species of the family Noctuidae.

\section{Results and discussion}

Lepidopteran caspases cluster into six distinct clades

As the genome of the silkworm Bombyx mori is the sole fully sequenced genome for insects of the order Lepidoptera to date, we first searched it for the presence of putative caspase genes (Figure 2). We identified a total of 5 genes, one on chromosome 4 (Caspase-3), three at various places on chromosome 10 (Caspase-1, -5 and -6) and one on chromosome 20 (Caspase-4) (Figure 2). Our findings are in agreement with a genome-wide analysis of the Bombyx mori genome for apoptosis-related genes, in which these 5 caspase genes have also been identified [37]. To get further insight into this gene family, we then mined publicly available as well as "in-house" EST datasets for transcripts encoding putative caspase enzymes. We retrieved a total of 66 transcripts corresponding to 63 different genes, from 27 species of moths and butterflies 


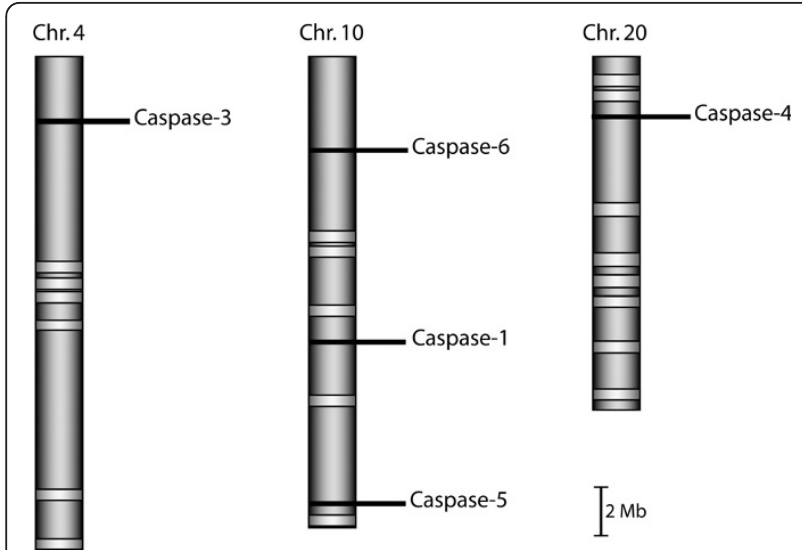

Figure 2 Schematic representation of the chromosomal localization of caspase genes on the Silkworm genome. The keyword "peptidase C14" was used to perform a search on the Kaikobase keyword search engine (http://sgp.dna.affrc.go.jp/ KAlKObase/). Putative gene sequences were retrieved and checked for conserved caspase domains.

spanning 11 lepidopteran families (Additional file 1). Caspase sequences were accepted if they fulfilled the following criteria: presence of the characteristic binding site $\left({ }^{\mathrm{L}} / \mathrm{s} / \mathrm{T}\right.$ $\mathrm{H}$ G) and/or active site (QAC $/ \mathrm{Q} G$ ), and level of amino acid identity (for complete amino acid alignments, see Additional files 2, 3, 4, 5, 6 and 7). A Bayesian inferred phylogenetic analysis clustered these sequences into six distinct clades (Figure 3), revealing an extra caspase gene family that was not found in the $B$. mori genome (Caspase-2). This observed number of caspase genes in Lepidoptera is similar to the seven genes found in Drosophila species: Drice, Dcp-1, Decay, Damm, Dronc, Dredd and Strica (also known as Dream) [13].

To facilitate our analysis at this stage, we decided to establish a naming convention for these genes based on the results of the phylogenetic analyses. The first caspase fully characterized for a lepidopteran insect has been named Caspase-1 preceded by the initials Sf, corresponding to the name of the species Spodoptera frugiperda [29]. We extended this convention by naming each caspase starting with the initials of the species from which it has been isolated, followed by "-Caspase" and by a number depending on which clade the sequence clustered into. Clades were arbitrarily numbered Caspase- 1 to Caspase-6. The term "Lep" was added to the cluster name to differentiate lepidopteran caspases from sequences derived from other organisms. When splicing variants were found (see below), we used the nomenclature proposed by Alnemri et al in which each variant is identified by a letter [38]. Here we stress the point that our nomenclature does not reflect any orthology between lepidopteran caspases and mammalian ones. For example, Lep-Caspase-2 is not an ortholog of Human caspase-2.

\section{Classification of the lepidopteran caspases}

The phylogenetic analysis clustered Lep-Caspase-1, -2 and -3 with Dcp-1, Drice and Decay (Figure 3). These 3 Drosophila caspases have been classified as effectors due to their short prodomain, their substrate specificity toward the tetrapeptide DEVD and their ability to cleave the Poly (ADP-ribose) polymerase and/or p35 [39-42]. Lep-Caspase- 1 and -3 also harbor a short prodomain, characteristic of effector caspases (Figure 4). However, no suitable cleavage site between the prodomain and the large subunit could be found in Lep-Caspase-2, and an amino acid alignment of Lep-Caspase- 1 and -2 sequences suggests that the latter lacks a prodomain (Additional file 8). Caspase-1 has already been characterized in S. frugiperda and S. littoralis. These studies demonstrated substrate specificity toward DEVD [30] and the ability to cleave p35 similar to the Drosophila effector caspases [29]. Although Drice and Dcp-1 are highly similar in sequence, several studies have shown that Drice and not Dcp-1 is the main effector caspase in Drosophila [41,43]. A study comparing the effect of single and double mutants for Drice and Dcp-1 showed that these two caspases have an overlapping function [44]. The role of Decay has still to be clarified. Finally, based on another phylogenetic analysis including all the known mosquito caspase sequences [45], we find no clear orthology between lepidopteran and dipteran effector caspases, which is due to the rapid evolution and diversity of this gene family (Additional file 9).

Like the Drosophila Dronc, to which it is closely related, and human Caspase-9, Lep-Caspase-5 harbors a CARD domain within its long prodomain (Figure 4). Typical CARD domains are constituted by a bundle of 6 alpha helices. An amino acid alignment of the prodomain of CARD containing caspases, including B. mori caspase5 , shows a low amino acid conservation, but a high conservation of the position of the 6 alpha helices (additional file 10). CARD domains are involved in the interaction of initiator caspases with Ark/Apaf1, to form the apoptosome which is required for the activation of Dronc/ human Caspase-9 $[46,47]$. It has been shown that dronc is ubiquitously expressed during the development of Drosophila and that its expression is stimulated by ecdysone during metamorphosis [22]. Lep-Caspase-6 is closely related to Drosophila Dredd. The presence, in the long prodomain, of 2 motifs composed of 6 alpha helices, forming a three-dimensional structure characteristic of the Death Domain family (Figure 4), strongly supports its classification as an initiator caspase [24]. An amino acid alignment of $B$. mori caspase- 6 with other Death Domain-containing caspases from human and Diptera shows again a low amino acid conservation, but the position of the alpha helices is conserved (Additional file 11). Despite the first description of Dredd as a potential 


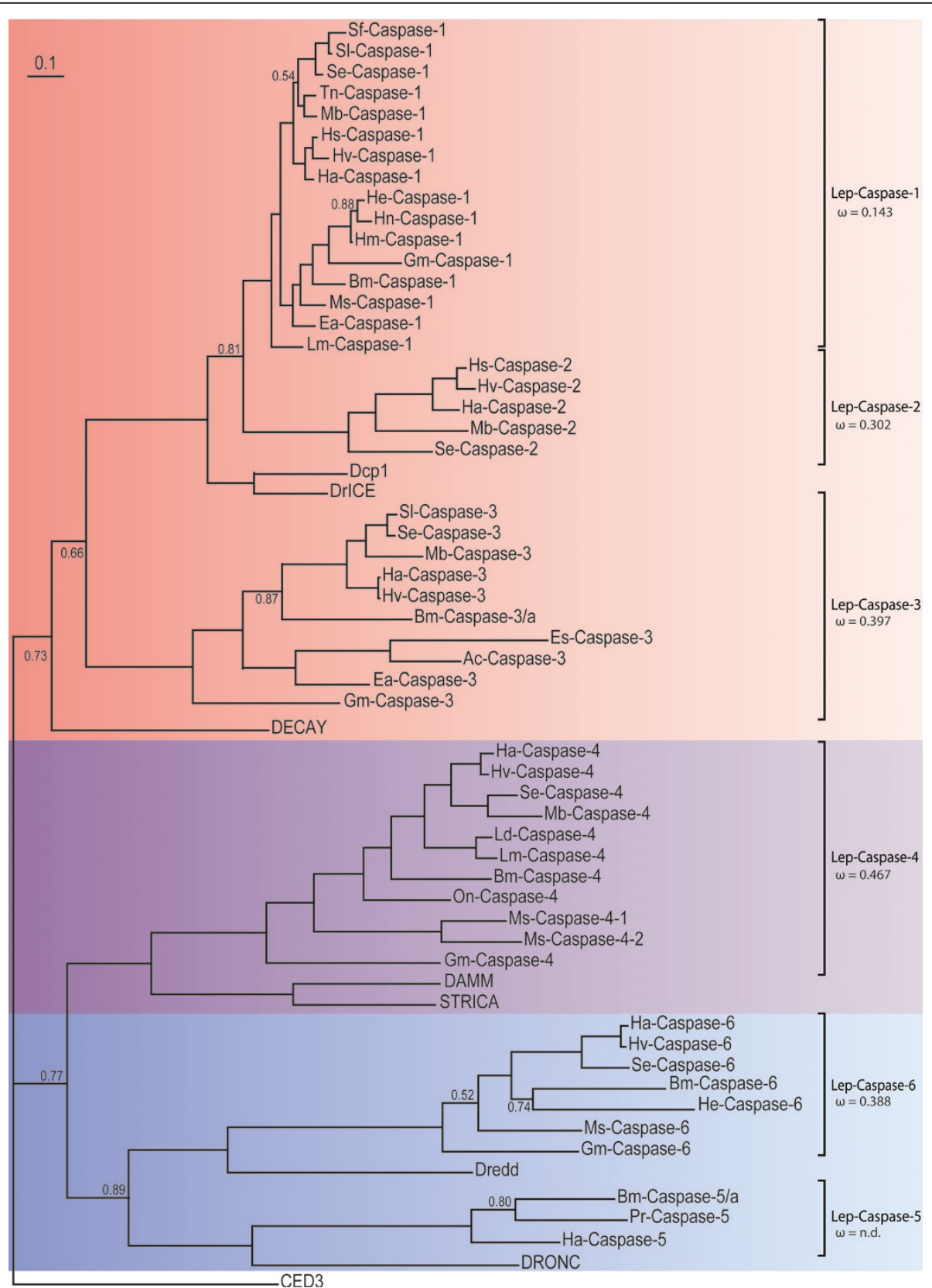

Figure 3 Phylogenetic relationships between caspase sequences found in Lepidoptera in relation with their Drosophila counterparts.

Amino acid alignments of caspase sequences without their prodomain were used to build a phylogenetic tree using a Bayesian inferred method with the C. elegans caspase Ced3 as an outgroup. Lepidopteran caspase clades were determined by branching patterns and are represented by brackets on the right. Posterior probabilities are shown only for nodes below 0.9 . $\mathrm{dN} / \mathrm{dS}$ ratio $(\omega)$ for each clade is mentioned below the clade name, except for Lep-Caspase- 5 for which the number of sequences was too low to determine it.

initiator caspase [48], it now appears to be more important in activating the innate immune response upon infection by Gram negative bacteria [49]. Based on a phylogenetic analysis including all the known mosquito caspase sequences, it is clear that Lep-Caspase- 5 and Lep-Caspase- 6 are the orthologs of dipteran Dronc and Dredd, respectively (Additional file 9).
Despite a relatively long prodomain, Lep-Caspase-4 does not harbor any known Death Domain which would have supported its classification as an initiator caspase (Figure 4). The phylogenetic analyses show a relationship with the Drosophila caspases Damm and Strica, for which the function has not been clearly defined yet. Lepidopteran Caspase-4 sequences share about 20\% 


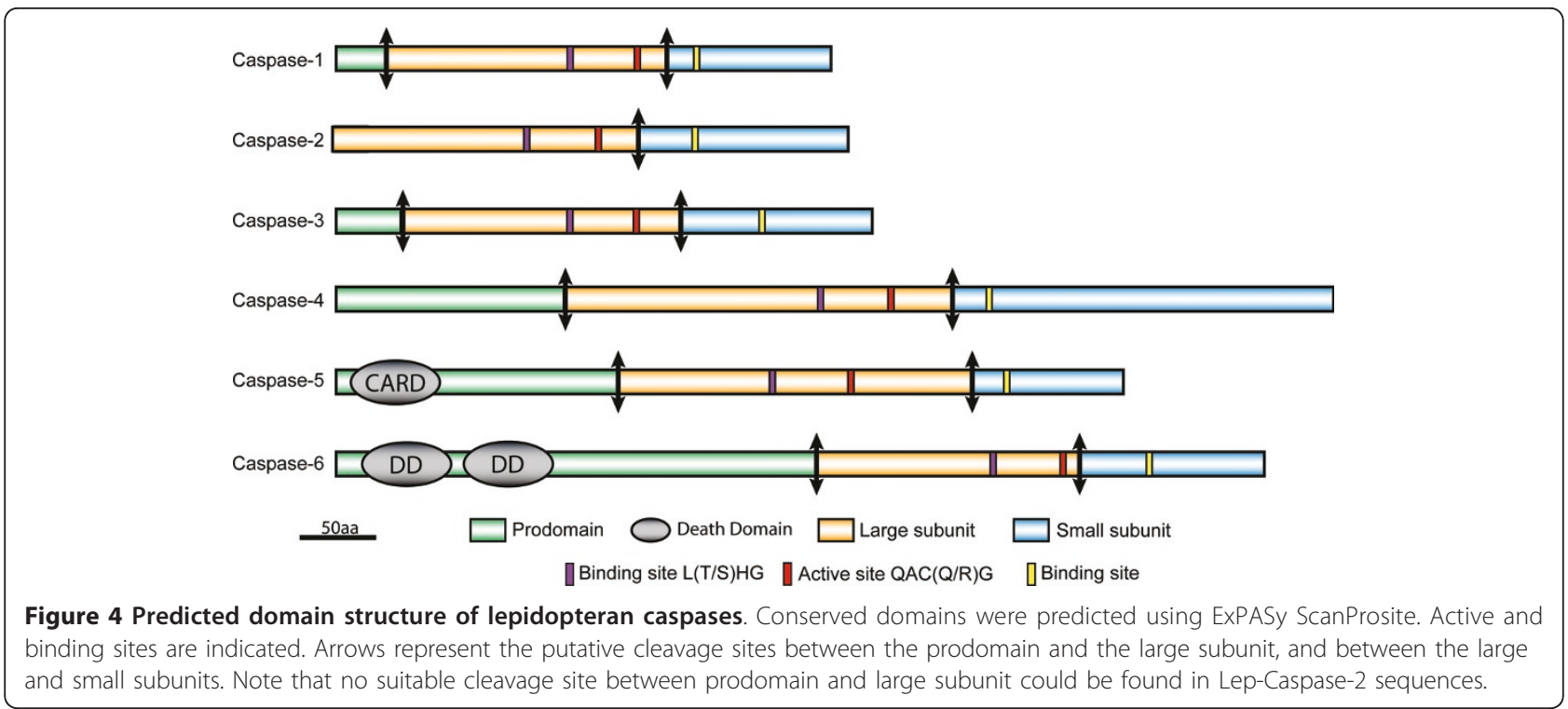

amino acid identity with Strica/Damm homologs from flies and mosquitoes, but do not harbor any serinethreonine-rich regions in the prodomain [50]. Although Damm and Strica are very similar in sequence, they appear to play different roles in D. melanogaster: Damm seems to be an effector caspase [51], whereas Strica seems to be an initiator caspase, exhibiting redundant activity with Dronc during oogenesis based on genetic studies [52]. Within the 12 sequenced Drosophila species, Damm is a more recent duplication of the ancestral Strica, as Damm is restricted to the melanogaster and obscura groups [45]. Perhaps the ancestral Strica was an initiator caspase in Diptera. Another unusual feature of Lep-Caspase-4 is its unique small subunit. It is twice as long as any other small subunit described so far, which usually ends 60 to 80 amino acids after the conserved serine residue $\left(\mathrm{Ser}^{428}\right.$ ) involved in substrate binding (Additional files 2, 3, 4, 5, 6 and 7). In addition, alignments (Additional file 5) show that the sequence corresponding to the last 140 residues of the small subunit is more variable compared to the rest of the subunit. However some residues are conserved in all sequences, including 3 cysteine residues potentially involved in the formation of secondary structures. These features indicate that Lep-Caspase- 4 is a peculiar caspase, the function of which cannot be solely assessed by sequence comparisons.

\section{Alternative splicing of Caspase- 3 and -5 in B. mori}

In $B$. mori, two isoforms of Caspase- 5 have been identified, a long form $(-5 / a)$ and a short form $(-5 / b)$ in which the exon encoding the sequence corresponding to the catalytic site is spliced out (See Additional file 12 for the intron-exon structure of lepidopteran caspases). The short form still possesses an intact prodomain and small subunit. In human, Caspase-9 splicing variants have been identified. In one of them, Caspase-9S, the catalytic site is missing and this isoform has been shown to be a dominant-negative inhibitor of apoptosis, by blocking the Caspase-9/Apaf1 interaction [53]. Enzymatically inactive caspase homologs that have arisen by gene duplication events have been recently described in Diptera [45]. These caspase-like decoy molecules may have acquired the ability to regulate other caspases. The short form of Bm-caspase- 5 might have evolved a similar decoy function by alternate splicing, however we could not find any separate genes encoding caspase-like decoy molecules in our analyses. Although several isoforms of Caspase- 5 were observed in B. mori, only a single transcript could be detected in $H$. armigera. In addition, the sequences corresponding to exon 6 and 7 in $B$. mori are part of a single exon in $H$. armigera, so a product like B. mori Caspase-5b could not be produced by alternate splicing in the latter species.

Three splicing variants were detected for Bm-Caspase-3 (Additional files 4 and 12), all of them harboring both catalytic and binding sites. Alternative splicing was not observed for Ha-Caspase-3. It has been suggested that catalytically active isoforms may differ in specificity and efficiency, which could fine-tune caspase activity through hetero-dimerization, resulting in amplification or inhibition of apoptosis [54].

\section{A noctuid-specific caspase gene arose from duplication of caspase-1}

Thorough mining of lepidopteran EST datasets for putative Caspase-2 led us to find the corresponding genes only in species of the family Noctuidae (Figure 3 and 
Additional file 1). No ortholog of Caspase-2 could be found in the silkworm genome (Figure 2). Taking into account that the amino acid identity between Lep-Caspase- 1 and Lep-Caspase- 2 sequences is relatively high (ranging between 47 and 53\%), we hypothesized that both genes may have arisen, in Noctuidae, from a tandem gene duplication event. To test this hypothesis, we screened a $H$. armigera $\mathrm{BAC}$ library using probes designed according to either the Ha-Caspase-1 or the Ha-Caspase-2 sequence. A BAC clone (clone P3E13, Genbank accession HQ645847) hit by both probes was identified and selected for sequencing. The Caspase- 1 and -2 genes were found, in a $43 \mathrm{~kb}$ fragment of this BAC, to be organized in tandem, separated by only $3 \mathrm{~kb}$ of non-coding DNA, were in the same orientation (Figure 5) and had no intron (Additional file 12), confirming our hypothesis. We then compared the organization of the genes surrounding Caspase- 1 between $H$. armigera and B. mori (Figure 5), we found a high degree of micro-synteny in this region of the genome between the two species, similar to what has been reported in recent studies $[55,56]$.

To examine the relative rates of sequence evolution within the Caspase- 1 and -2 subfamilies since divergence, pairwise comparisons of sequences obtained from noctuid species were performed by applying the Nei-Gojobori algorithm [57] (see Additional file 13 for the P-distances of synonymous and non-synonymous substitutions). Mean of synonymous substitutions per site $\left(\mathrm{d}_{\mathrm{S}}\right)$ was 0.480 and 0.549 within Caspase- 1 and Caspase- 2 respectively; mean $\mathrm{d}_{\mathrm{S}}$ between Caspase- 1 and -2 is 0.646 , indicating saturation. Mean non-synonymous substitutions per site $\left(\mathrm{d}_{\mathrm{N}}\right)$ was 0.047 for Caspase- 1 and 0.170 for Caspase-2. We used the Codon-based Z-test [58] to test the null hypothesis $\mathrm{d}_{\mathrm{S}}$ $=\mathrm{d}_{\mathrm{N}}$ which would indicate that both genes are neutrally evolving. We could reject this null hypothesis as we found that $d_{N}$ is significantly smaller than $d_{S}$ (Caspase- $1: Z=$ -17.000, $\mathrm{p}<0.001$; Caspase-2: $\mathrm{Z}=-14.138, \mathrm{p}<0.001$ ), indicating a strong purifying selection on both genes. We also tested whether synonymous and non-synonymous substitution rates differ between the two genes $\left(\mathrm{d}_{\text {SCasp } 1}=\right.$ $\mathrm{d}_{\mathrm{SCasp} 2}$ and $\mathrm{d}_{\mathrm{NCasp} 1}=\mathrm{d}_{\mathrm{NCasp} 2}$, same Z-test as before) we could not reject that $\mathrm{d}_{\text {SCasp } 1}=\mathrm{d}_{\text {SCasp } 2}(\mathrm{Z}=-2.03, \mathrm{p}>0.05)$, but the non-synonymous substitution rate in Caspase- 2 was significantly higher compared to the one in Caspase-1 $(\mathrm{Z}=-8.853, \mathrm{p}<0.001)$. The coefficient of functional divergence [59], which measures the overall difference between site-specific substitutions among the two subfamilies is $\theta=$ 0.57 , which is significantly greater than zero (likelihood ratio test, $\chi^{2}=7.31, \mathrm{P}<0.01$ ). These results strongly suggest that Caspase-2, though also under purifying selection following the duplication event, has evolved under much more relaxed selective constraints than Caspase-1. It has been well documented that gene duplication is often followed by a period of relaxed selective constraints on one of the duplicate, allowing it to accumulate more mutations $[60,61]$. Although it has been suggested that gene duplication occurs at a high rate of $0.01 /$ gene/million years, the most common fate of duplicated genes is silencing and loss [62]. In some cases however, the duplicate is retained as functional gene and either a new function is acquired by one of the copies or the ancestral function is maintained or subdivided between the two copies [63]. The fact that the cleavage site differ between the two subunits (TETD for Caspase-1 and xETD for Caspase-2), and that Caspase- 2 may lack a prodomain, suggests that they might be activated by different signals. This could indicate a subfunctionalization of Caspase-2 in Noctuidae.

\section{Ms-Caspase-4 subfamily has evolved through duplication events}

Several contigs corresponding to putative Caspase- 4 transcripts were identified from an EST dataset derived from a $M$. sexta larval midgut cDNA library, sequenced by 454 pyrosequencing [64]. Analyses of complete sequences

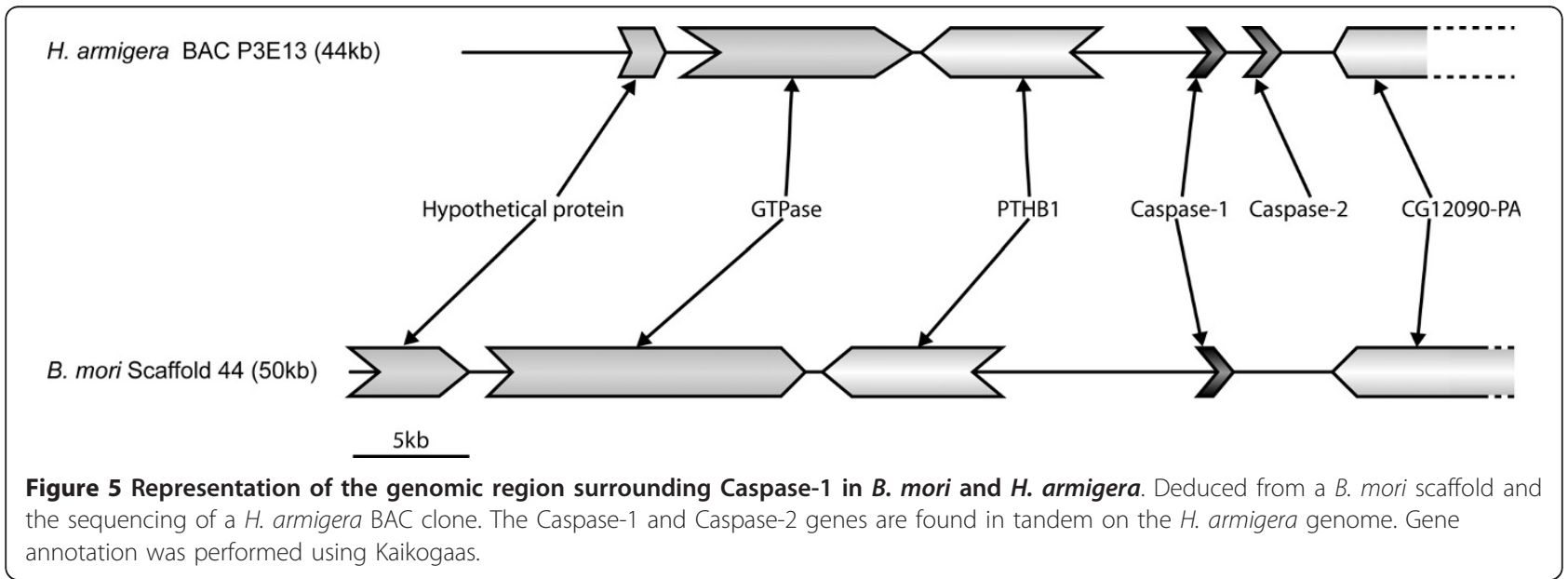


obtained by RACE-PCR resulted in 3 distinct cDNAs. Two of them, Ms-Caspase-4-1/a and -4-1/b, differ only by 20 single nucleotide polymorphisms (SNPs), 8 of which are non-synonymous, as well as an extra codon encoding $\mathrm{ASN}^{113}$ in Ms-Caspase-4-1/b (Figure 6A). The third sequence, Ms-Caspase-4-2, encodes a protein sharing $\sim 63 \%$ amino acid identity with both Ms-Caspase-4-1/ $\mathrm{a}$ and $-4-1 / \mathrm{b}$ (Figure 6A). The occurrence of several unique transcripts for Lep-Caspase-4 has only been found in that species so far. A closer look at the region where Bm-Caspase- 4 is located on the silkworm genome did not reveal any extra Lep-caspase-4 genes or even pseudogenes nearby Bm-Caspase-4 (Figure 6B). These results suggest that Caspase- 4 has undergone at least two duplication events in $M$. sexta. A phylogenetic study of dipteran caspases suggest that Damm and Strica, the homologs of Lep-Caspase-4, have arisen by duplication in the melanogaster and obscura clades, while only Strica is present in the other drosophilid clades [45]. In mosquitoes, the ancestor of Damm/Strica has undergone many duplication events after the divergence of the Anopheles gambiae, Culex quinquefasciatus and Aedes aegypti

\section{A $\begin{array}{llllllllllll}10 & 20 & 30 & 40 & 50 & 60 & 70 & 80 & 90 & 100\end{array}$ \\ Bm-Caspase-4 Ms-Caspase-4-1/a Ms-Caspase-4-1/b Ms-Caspase-4-2 \\ Bm-Caspase-4 Ms-Caspase-4-1/a Ms-Caspase-4-1/b Ms-Caspase-4-2 \\ Bm-Caspase-4 Ms-Caspase-4-1/a Ms-Caspase-4-1/b Ms-Caspase-4-2 \\ Bm-Caspase-4 Ms-Caspase-4-1/a Ms-Caspase-4-1/b Ms-Caspase-4-2 \\ Bm-Caspase-4 Ms-Caspase-4-1/a Ms-Caspase-4-1/b Ms-Caspase-4-2 \\ Bm-Caspase-4 Ms-Caspase-4-1/a Ms-Caspase-4-1/b Ms-Caspase-4-2

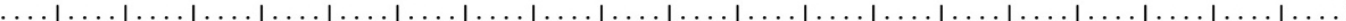 MDS--DENQLQTNSKTKNKRKKYLKRSKE--- MDSELQDTSLLQPETELKKKRKRR-NRGKQTRMSDDVDNGSSDSNVVEADALDNANSEE I IDLMTNKWE IDDSPRVNNDINENTEYYETPIDTIHSQNPS MDSELQDTSLQKTETELKKKRKRR-NRGKQTRMSDDVDNGSSDSNVVEADALDNANTEE I IDLMTNKWE IDDSPRVNNDINENTEYYETSIDT IHSQNPS MEGENKGTNIQNQGTDLQKKRKRK-KRGKQKRISEDVDNGSCVSNIVELDAPDNANTVENIDLITNKWEIDENPRFDKNTNENTEYHEATTDTLHSQSSS $\begin{array}{cccccccccc}110 & 120 & 130 & 140 & 150 & 160 & 170 & 180 & 190 & 200\end{array}$ . (1) RVETGQRFFSDA-NDD--EYYRRKDANLTADNNVNIFTGVSQKTNNVFTTYDGIASSCVTSNENITKMEKCSITRLTSEDVEKLYPSTSSATETVPQL-- RVETGQRFF SDANNDD--EYYRRKDANLTADNNVNIFTGVSQKTNNVFTTYDGIASSCVTSNENITKMEKSSITRLTSEDVEKLYPSTSSATETVPQL-- QVKTCEHLFSYSNNND PPDYEHLKDENLSADIKNNIV------VQNLVT--DGIPSTCENPSTKVLHDGNKLSTQLSSESDTKLNRSISSTTKNLIQL-- \\ $\begin{array}{llllllllll}210 & 220 & 230 & 240 & 250 & 260 & 270 & 280 & 290 & 300\end{array}$ $\ldots|\ldots| \ldots|\ldots| \ldots|\ldots| \ldots|\ldots| \ldots|\ldots| \ldots|\ldots| \ldots|\ldots| \ldots|\ldots| \ldots|\ldots| \ldots|\ldots| \ldots|\ldots| \ldots|\ldots| \ldots|\ldots|$ ESKFYNNRALTKYATTYELEKFEKNALLIFNQENIYGYSPRKGTEKDVESLTVTFQKFGFEVVEHKDLTKDEVLNEIKEFSSRDLRDYGCIGIAVLTHGS ---YNARALPKEAKTYELENFDRNVLI I FNQKD-FGTHQRLGTDEDVKALKKTFETFNFDVLPYTDRTVAQIKEILDAISCQDFSDYGCVAIAVLTHGD ----YNARALPKEAKTYELENFDRNVLI IFNQKD-FGTHQRLGTDQDVKALKKTFEAFNFDVLPYTDRTVAQIKE ILDAISCQDFSDYGCVAIAVLTHGD ----YNPRALPKESETYELEKFDKNKLI IFNQIK-FGQYERLGTEADLKSLKTTFEAFNFEVQDYPDPTVDKIEEILNEISVTDYSDYGCLAIAVLTHGG \\ $\begin{array}{llllllllll}310 & 320 & 330 & 340 & 350 & 360 & 370 & 380 & 390 & 400\end{array}$ $\ldots|\ldots| \ldots|\ldots| \ldots|\ldots| \ldots|\ldots| \ldots|\ldots| \ldots|\ldots| \ldots|\ldots| \ldots|\ldots| \ldots|\ldots| \ldots|\ldots| \ldots|\ldots| \ldots \mid$ SDGMLRAKDMQY SEKE ILRHFKVHDKPFLVTKPKFLI IQACRGKEEMQGAAVFRS GKLRKDLDEDLEPYVLPIEADMLILHSSY SGKPSHRHEVDGSWFI IDGSLSAKDDDY PEMDI INTLKTHKNHSLITKPRLLFVQTCRGPNALAGVPVLRKGKVKSNLFYD SKPYALPVEADMLVVHSSYTGNRTHRHEVDGSWFI IDGSI SAKDDDYPEMDI INTLKTHKNHSLITKPRLLFVOACRGPNALAGVPVLRKGKVKSNLFYDSKPYTLPVEADMLVVHSSYTGNRTHRHEVDGSWE IDGKLLAADGDYYE IKI INFLKTHKNHSLITKPRLLFVQACRGPNSLIGVPVLQPGKVQSNLIYDTEPYTLPVEADMLVVHSSYVGNRAVRHQREGSWFI \\ $\begin{array}{rrrrrrrrrr}410 & 420 & 430 & 440 & 450 & 460 & 470 & 480 & 490 & 500\end{array}$ $\ldots|\ldots| \ldots|\ldots| \ldots|\ldots| \ldots|\ldots| \ldots|\ldots| \ldots|\ldots| \ldots|\ldots| \ldots|\ldots| \ldots|\ldots| \ldots|\ldots| \ldots|\ldots| \ldots|\ldots|$ QSLCSKINELSETHDLES I T TEVKREVAIDRYHKEFNRRT SEYDINKQMPVI TSTLIRKLYLRKYMDPPVSQCLIKPTPAISESSKNEVLDESTPDTNLL QTLCKKINEMGQTDDLETILTHIKHEVAIDKEHEVYNRVTGEYDINKQMPVVTTTLIRKLYLRR------DAAEGTVTSNTSEMPGAIAVDSHNAGANPE QTLCKKINEMGQTDDLETILTHIKHEVAIDKEHEVYNRVTGEYDINKQMPVVTTTLIRKLYLRR------DAAEGTVTSNTSEMPGAIAVDSHNAGANPE QTLCKKINEMASTHDLETILTHIKREVAIDKEKI IVNRVTKEHE INKQMPVVTTTLIRKLYLKR------SAAEENAL-NTNGMPTDVAVDSYNTGANPK

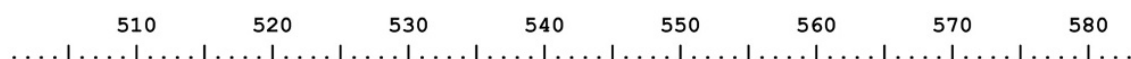

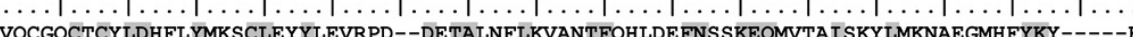 P----CLCTLDYYNYIRDCLREYVRDHDQ--DTTARSLLDLTKTFKDEETFNATKEQCALTIADHLEHSVKHYERYKFVYIKRI P----CLCTLDYYNY IRDCLREYVRDHDQ--DTTARSLLDLTKTFKDEETFNATKEQCALTIADHLEHSVKHYERYKFVY IKRI PELENCRCNLNYFDY I KECLRHFLQDNNDYKDTTGRSLLNLTDTLKNEEAFNATKEKCAMTIADYLETRAKHCQHYKYIYINKK}

B

B. mori Scaffold $37(150 \mathrm{~kb})$
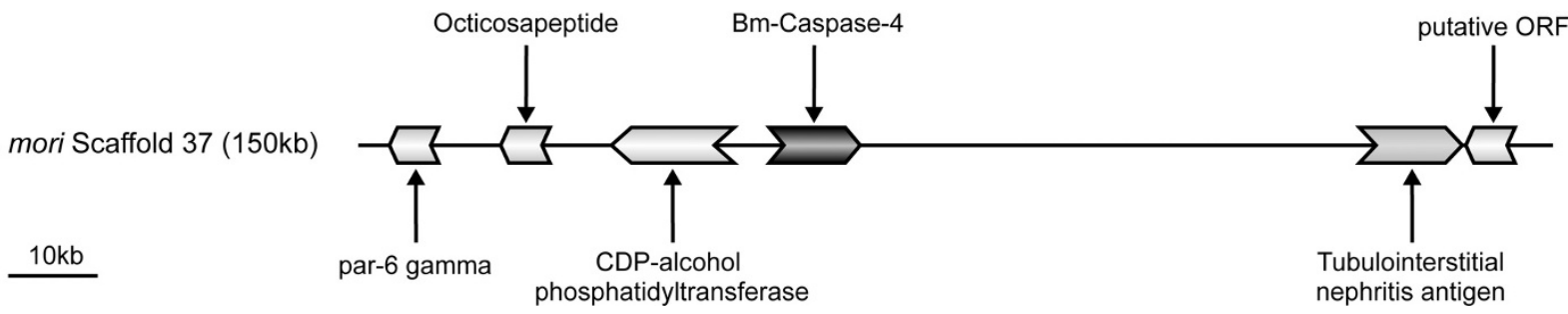

par-6 gamma

phosphatidyltransferase

Tubulointerstitial nephritis antigen

Figure 6 Recent gene duplications of the Caspase-4 gene in $\boldsymbol{M}$. sexta. (A) Amino acid alignment of the Caspase-4 sequences from B. mori and $M$. sexta. Identical amino acids are shaded in grey. (B) Schematic representation of the genomic region surrounding the Caspase-4 gene deduced from a B. mori scaffold. Gene annotation was performed using Kaikogaas. 
lineages. Two homologs of Damm/Strica have been described in A. gambiae, as well as 2 in C. quinquefasciatus and 4 in A. aegypti $[14,45]$. All of the duplicates are potentially active since they all harbor the critical amino acids involved in binding and active sites. However, no biochemical characterization has been carried out on these caspases so far. The paucity of EST data for members of the family Sphingidae does not allow us to conclude whether the duplication of Lep-Caspase-4 is specific only to $M$. sexta or if it has also happened in sister species.

\section{Conclusions}

Despite obvious limitations of EST datasets in term of available species and transcriptome coverage, we were able to identify 63 caspase genes coming from 27 different lepidopteran species. Based on the phylogenetic analyses, we showed that the lepidopteran caspase family is represented by at least 5 members. Nevertheless, the biochemical characterization of these caspases has to be performed to clarify the exact function and their potential interactions. The main shaping forces of the caspase gene family in mammals as well as in other insects, such as mosquitoes, are gene deletions and gene innovations by duplication events $[16,45]$. Although we cannot assess whether deletions of caspase genes has happened in Lepidoptera, our data suggest that gene duplication is one of the shaping forces of this gene family in these insects. Availability in the near future of whole genome sequences and transcriptomes from other species of the order Lepidoptera as well as from other insect orders, will improve our understanding of the complexity of this gene family and its evolutionary history in insects in general.

\section{Methods}

\section{Preparation of CDNA libraries and EST sequencing}

Generation of cDNA libraries for T. ni whole larvae [65]; Plutella xylostella, Pieris rapae, Colias eurytheme and Anthocharis cardamines whole larvae [66]; and for $H$. armigera midgut and S. littoralis whole larvae [55] were previously described. TRIzol Reagent (Invitrogen) was used to isolate total RNA from whole larvae or dissected larval tissues of Pontia daplidice, Eucheira socialis, Lymantria dispar, Lymantria monacha, G. mellonella, $H$. virescens, Mamestra brassicae, and Spodoptera exigua. Normalized, full-length, enriched cDNA libraries were generated using both the Creator SMART cDNA library construction kit (BD Clontech) and the Trimmer Direct cDNA normalization kit (Evrogen), generally following the manufacturer's protocol but with several important modifications. In brief, $2 \mu \mathrm{g}$ of poly(A)+ mRNA was used for each cDNA library generated. Reverse transcription was performed with a mix of several reverse transcriptases for $1 \mathrm{~h}$ at $42^{\circ} \mathrm{C}$ and 90 minutes at $50^{\circ} \mathrm{C}$. cDNA size fractionation was performed with SizeSep 400 spun columns (GE Healthcare) that resulted in a cutoff at 200 bp. The full-length, enriched cDNAs were cut with SfiI and ligated to the SfiI-digested pDNR-Lib plasmid vector (Clontech). Ligations were transformed into E. coli ELECTROMAX DH5 $\alpha$-E electro-competent cells (Invitrogen). Plasmids from bacterial colonies grown in 96 deep-well plates were prepared using the Robot 96 Plasmid isolation kit (Eppendorf) on a Tecan Evo Freedom 150 robotic platform (Tecan). Single-pass sequencing of the 5' termini of cDNA libraries was carried out on an ABI 3730 xl Automatic DNA Sequencer (PE Applied Biosystems). Vector clipping, quality trimming, and sequence assembly were done with the Lasergene 8 software package (DNAStar Inc.). BLAST searches were conducted on a local server using the National Center for Biotechnology Information (NCBI) blastall program. Sequences were aligned using ClustalW software [67].

\section{Database mining for putative caspase sequences}

The protein sequence of Sf-Caspase-1 (AF548387) [29] was used as a query to perform TBLASTN searches of lepidopteran EST databases publicly available on NCBI dbEST, on InsectaCentral $[68,69]$, or in-house (Additional file 1). Sequences were accepted as being "caspases" when presenting the characteristic binding site $\left({ }^{\mathrm{L}} / \mathrm{s} / \mathrm{T} / \mathrm{H} \mathrm{G}\right)$ and/or active site ( $\left(\mathrm{QC}^{\mathrm{R}} / \mathrm{Q} G\right)$. Some exceptions were made when partial amino acid sequences presented a strong amino acid identity with sequences previously accepted as caspases.

\section{Caspase genes amplification and sequencing}

Specific primers (Additional file 14) were designed in order to re-amplify BmCasp-4, and -5 , as well as HaCasp$1,-2,-3$ and -4 using total RNA prepared respectively from $B$. mori and $H$. armigera whole larvae as starting material. In order to get full length cDNA, 5'-and 3'-RACE were performed for HaCasp-5, and -6, BmCasp-4, MsCasp-1, -4-2 and -6 as well as EaCasp-1 and -3 using the SMART ${ }^{\circledR}$ RACE cDNA amplification kit (Clontech), following the manufacturer's instructions. Total RNA from $H$. virescens, $H$. subflexa and $H$. armigera larvae was extracted using TRIzol, according to the manufacturer's specifications. Forward and reverse specific primers designed according to the HvCasp- 6 sequence (Additional file 14) were used to amplify HaCasp-6 from $H$. armigera cDNAs. Forward and reverse specific primers designed according to the HaCasp-1 and - 2 sequences (Additional file 14) were used to amplify Casp-1 and -2 from $H$. virescens and $H$. subflexa cDNAs. Finally, a $H$. armigera draft genome assembly generated by 454 pyrosequencing (unpublished data) was searched using BmCasp- 5 as query to identify a homolog in $H$. armigera. Forward and reverse primers (Additional file 14) were designed based on the 
sequences of contigs 28382 and 105066 in order to amplify HaCasp- 5 sequence from cDNAs extracted from $H$. armigera HaAM1 cells [70] treated with $75 \mu \mathrm{M}$ ecdysone. All amplicons were then ligated in pCR2.1-Topo ${ }^{\mathbb{B}}$ vector (Invitrogen). Ligations were transformed into $E$. coli TOP10 chemically competent cells (Invitrogen) and plasmid mini-preparation and sequencing were performed as mentioned above.

\section{Sequence alignment and phylogenetic analysis}

Deduced amino acid sequences from caspase transcripts retrieved from the various databases searched were aligned together with the Drosophila caspases (Drice, Dcp-1, Decay, Dronc, Dredd, Damm and Strica) and with the $C$. elegans caspase Ced3 as an outgroup, using the FFT-Ns-i strategy implemented in the MAFFT alignment program [71]. After removing the prodomain, the amino acid alignment was then used for the phylogenetic analyses. Only sequences containing the complete open reading frame (ORF) or partial sequences containing at least the part encoding the complete large and small subunits were used to build the phylogenies. The phylogenetic reconstruction was done by Bayesian inference using MrBayes 3.1 [72]. The prior was set for the amino acid model to mix, thereby allowing model jumping between fixed-rate amino acid models. Markov Chain Monte Carlo runs were carried out for 10,000,000 generations after which log likelihood values showed that equilibrium had been reached after the first 400 generations in all cases, and those data were discarded from each run and considered as 'burnin'. Two runs were conducted for the dataset showing agreement in topology and likelihood. To determine which clusters partial caspase sequences belong to, amino acid sequences were aligned with MAFFT using the FFT-Ns-i strategy; and a phylogenetic tree was generated by Neighbor joining (1000 replicates) using MEGA4 software [73]. Relative-rate tests were applied to compare rates of evolution in the Caspase- 1 and Caspase- 2 subfamilies. Synonymous and non synonymous substitution rates were determined by the Nei and Gojobori algorithms [57] as implemented in MEGA4. Statistics were performed using the Codon-based Z-test, which corresponds to a t-test with an infinite degree of freedom [74]. The coefficient of functional divergence $\theta$ was calculated using the program DIVERGE 2.0 [75]. This coefficient ranges between 0 and 1 and measures the overall degree to which site-specific substitution rates differ; values significantly greater than zero indicate rate differences among homologous sites in the two subfamilies. In order to characterize the primary structure and domain architecture of the different caspases, their predicted amino acid sequences were submitted to ExPASy ScanProsite [76]. For comparison to dipteran caspases, sequences from $D$. melanogaster, Culex quinquefasciatus, Anopheles gambiae, and Aedes aegypti were extracted from Supplementary Figure S1 of ref [45].

\section{BAC library screening and sequencing}

Nylon filters from a Helicoverpa armigera BAC library established from the strain Toowoomba were washed, blocked, and hybridized with horseradish peroxidaselabelled DNA fragment containing part of the $H$. armigera Casp-1 and Casp-2 genes. Labeling, hybridization, and probe detection were done according to specifications in the ECL DNA labeling and detection kit (GE Healthcare). Positive clones were isolated from glycerol stocks, grown in Terrific Broth, and BAC DNA was isolated with the Nucleobond Xtra Maxi Kit according to the manufacturer's instructions (Macherey-Nagel). BAC genomic DNA quantities were estimated spectrophotometrically on a Nanodrop ND 1000 (Peqlab biotechnologie $\mathrm{GmbH}$ ). Positive clones were digested by EcoR1 and HindIII, blotted and re-hybridized first with $\mathrm{Ha}-$ Caspase- 1 probe then with Ha-Caspase- 2 to identify positives clones for the 2 probes. BAC DNA was sheared into two different size ranges (1.5 -2 kb and 4-5 $\mathrm{kb}$ ) with a hydroshear device (Molecular devices), blunted with the Quick blunting kit (New England Biolabs), isolated from agarose gel, column purified, and ligated into the pUC19-SmaI vector (Fermentas). Ligations were transformed into E. coli ELECTROMAX DH5 $\alpha$-E electrocompetent cells (Invitrogen). Plasmid preparation, sequencing, and assembly were performed as mentioned above.

\section{Intron-Exon structure}

Bombyx mori caspase cDNA sequences were compared to the genomic sequences retrieved from KAIKObase $[77,78]$ (Caspase-1: BGIBMGA006940, Caspase-3: BGIBMGA 006131, Caspase-4: BGIBMGA004420, Caspase-5: BGIBMGA002841, Caspase-6: BGIBMGA006726) using the Spidey software available at NCBI. The same comparison was done using $H$. armigera cDNA sequences and the genomic sequences retrieved from the $H$. armigera draft genome assembly mentioned above.

\section{Additional material}

Additional file 1: Table S1. Details of the caspase sequences characterized in this study.

Additional file 2: Figure S1. Amino acid alignment of Lep-Caspase-1 sequences.

Additional file 3: Figure S2. Amino acid alignment of Lep-Caspase-2 sequences.

Additional file 4: Figure S3. Amino acid alignment of Lep-Caspase-3 sequences.

Additional file 5: Figure S4. Amino acid alignment of Lep-Caspase-4 sequences. 
Additional file 6: Figure S5. Amino acid alignment of Lep-Caspase-5 sequences.

Additional file 7: Figure S6. Amino acid alignment of Lep-Caspase-6 sequences.

Additional file 8: Figure S7. Amino acid alignment of noctuid-derived caspase- 1 and caspase-2 sequences.

Additional file 9: Figure S8. Phylogenetic relationship of caspases sequences found in Lepidoptera and Diptera.

Additional file 10: Figure S9. Alignment of Bm-Caspase-5 with the prodomains of Drosophila Dronc, Aedes aegypti Ae-Dronc and human Caspase-1, -2 and -9 .

Additional file 11: Figure S10. Alignment of Bm-Caspase- 6 with the prodomains of Drosophila Dredd, Aedes aegypti Ae-Dredd and human Caspase-8 and -10.

Additional file 12: Figure S11. Intron-exon structure of lepidopteran caspases.

Additional file 13: Figure S12. P-distances of synonymous and nonsynonymous substitutions among the Noctuids caspase-1 and -2 .

Additional file 14: Table S2. Primer sequences used for amplification of caspase transcripts.

\section{Acknowledgements}

We are particularly grateful to D. Schnabelrauch and H. Ringys-Beckstein for providing technical assistance for sequencing. We thank Hanna HeidelFischer for helpful comments on the manuscript and her help with MrBayes. This work was supported by the Max-Planck-Gesellschaft.

\section{Authors' contributions}

JC, YP and DGH conceived the study. JC, YP and HV performed experiments. $J C, Y P$ and HV analyzed the data. JC and YP wrote the initial manuscript and all authors contributed to the preparation of the final version. All authors read and approved the final manuscript.

\section{Competing interests}

The authors declare that they have no competing interests.

Received: 23 February 2011 Accepted: 8 July 2011

Published: 8 July 2011

\section{References}

1. Penaloza C, Orlanski S, Ye Y, Entezari-Zaher T, Javdan M, Zakeri Z: Cell death in mammalian development. Curr Pharm Des 2008, 14:184-196.

2. Amundson SA, Myers TG, Fornace AJ Jr: Roles for p53 in growth arrest and apoptosis: putting on the brakes after genotoxic stress. Oncogene 1998, 17:3287-3299.

3. Creagh EM, Conroy H, Martin SJ: Caspase-activation pathways in apoptosis and immunity. Immunol Rev 2003, 193:10-21.

4. Lockshin RA, Williams CM: Programmed cell death-II. Endocrine potentiation of the breakdown of the intersegmental muscles of silkmoths. Journal of Insect Physiology 1964, 10:643-649.

5. Weeks JC, Truman JW: Independent steroid control of the fates of motoneurons and their muscles during insect metamorphosis. J Neurosci 1985, 5:2290-2300

6. Lockshin RA, Zakeri Z: Programmed cell death: early changes in metamorphosing cells. Biochem Cell Biol 1994, 72:589-596.

7. Truman JW: Cell death in invertebrate nervous systems. Annu Rev Neurosci 1984, 7:171-188.

8. Uwo MF, Ui-Tei K, Park P, Takeda M: Replacement of midgut epithelium in the greater wax moth, Galleria mellonela, during larval-pupal moult. Cell Tissue Res 2002, 308:319-331.

9. Parthasarathy R, Palli SR: Developmental and hormonal regulation of midgut remodeling in a lepidopteran insect, Heliothis virescens. Mechanisms of Development 2007, 124:23-34

10. Clem RJ: Baculoviruses and apoptosis: a diversity of genes and responses. Curr Drug Targets 2007, 8:1069-1074.
11. Koonin EV, Aravind L: Origin and evolution of eukaryotic apoptosis: the bacterial connection. Cell Death Differ 2002, 9:394-404.

12. Shaham S: Identification of Multiple Caenorhabditis elegans Caspases and Their Potential Roles in Proteolytic Cascades. Journal of Biological Chemistry 1998, 273:35109-35117.

13. Kumar S, Doumanis J: The fly caspases. Cell Death Differ 2000, 7:1039-1044.

14. Bryant $\mathrm{B}$, Blair CD, Olson KE, Clem RJ: Annotation and expression profiling of apoptosis-related genes in the yellow fever mosquito, Aedes aegypti. Insect Biochem Mol Biol 2008, 38:331-345.

15. Chowdhury I, Tharakan B, Bhat GK: Caspases-An update. Comparative Biochemistry and Physiology Part B: Biochemistry and Molecular Biology 2008, 151:10-27

16. Eckhart L, Ballaun C, Hermann M, VandeBerg JL, Sipos W, Uthman A Fischer H, Tschachler E: Identification of Novel Mammalian Caspases Reveals an Important Role of Gene Loss in Shaping the Human Caspase Repertoire. Mol Biol Evol 2008, 25:831-841.

17. Robertson AJ, Croce J, Carbonneau S, Voronina E, Miranda E, McClay DR, Coffman JA: The genomic underpinnings of apoptosis in Strongylocentrotus purpuratus. Developmental Biology 2006, 300:321-334.

18. Ameisen JC: On the origin, evolution, and nature of programmed cell death: a timeline of four billion years. Cell Death Differ 2002, 9:367-393.

19. Talanian RV, Quinlan C, Trautz S, Hackett MC, Mankovich JA, Banach D, Ghayur T, Brady KD, Wong WW: Substrate specificities of caspase family proteases. J Biol Chem 1997, 272:9677-9682

20. Garcia-Calvo M, Peterson EP, Rasper DM, Vaillancourt JP, Zamboni R, Nicholson DW, Thornberry NA: Purification and catalytic properties of human caspase family members. Cell Death Differ 1999, 6:362-369.

21. Nicholson DW: Caspase structure, proteolytic substrates, and function during apoptotic cell death. Cell Death Differ 1999, 6:1028-1042.

22. Dorstyn L, Colussi PA, Quinn LM, Richardson H, Kumar S: DRONC, an ecdysone-inducible Drosophila caspase. Proc Natl Acad Sci USA 1999, 96:4307-4312.

23. Fuentes-Prior P, Salvesen GS: The protein structures that shape caspase activity, specificity, activation and inhibition. Biochem J 2004, 384:201-232

24. Park HH, LO YC, Lin SC, Wang L, Yang JK, Wu H: The death domain superfamily in intracellular signaling of apoptosis and inflammation. Annu Rev Immunol 2007, 25:561-586.

25. Chang $\mathrm{HY}$, Yang $X$ : Proteases for cell suicide: functions and regulation of caspases. Microbiol Mol Biol Rev 2000, 64:821-846.

26. Fan TJ, Han LH, Cong RS, Liang J: Caspase family proteases and apoptosis. Acta Biochim Biophys Sin (Shanghai) 2005, 37:719-727.

27. Dorstyn L, Mills K, Lazebnik Y, Kumar S: The two cytochrome c species, DC3 and DC4, are not required for caspase activation and apoptosis in Drosophila cells. The Journal of Cell Biology 2004, 167:405-410.

28. Clem RJ, Fechhmeimer M, Miller LK: Prevention of apoptosis by a baculovirus gene during infection of insect cells. Science 1991, 254:1388-1390

29. Ahmad M, Srinivasula SM, Wang L, Litwack G, Fernandes-Alnemri T, Alnemri ES: Spodoptera frugiperda caspase-1, a novel insect death protease that cleaves the nuclear immunophilin FKBP46, is the target of the baculovirus antiapoptotic protein p35. J Biol Chem 1997, 272:1421-1424

30. Liu Q, Qi Y, Chejanovsky N: Spodoptera littoralis caspase-1, a Lepidopteran effector caspase inducible by apoptotic signaling Apoptosis 2005, 10:787-795

31. Yang D, Chai L, Wang J, Zhao X: Molecular cloning and characterization of Hearm caspase-1 from Helicoverpa armigera. Mol Biol Rep 2007.

32. Hebert CG, Valdes JJ, Bentley WE: Investigating apoptosis: characterization and analysis of Trichoplusia ni-caspase-1 through overexpression and RNAi mediated silencing. Insect Biochem Mol Biol 2009, 39:113-124.

33. Manji GA, Friesen PD: Apoptosis in motion. An apical, P35-insensitive caspase mediates programmed cell death in insect cells. J Biol Chem 2001, 276:16704-16710.

34. Zoog SJ, Schiller JJ, Wetter JA, Chejanovsky N, Friesen PD: Baculovirus apoptotic suppressor P49 is a substrate inhibitor of initiator caspases resistant to P35 in vivo. Embo J 2002, 21:5130-5140.

35. Seshagiri S, Miller LK: Baculovirus inhibitors of apoptosis (IAPs) block activation of Sf-caspase-1. Proc Natl Acad Sci USA 1997, 94:13606-13611.

36. Xia QY, Wang J, Zhou ZY, Li RQ, Fan W, Cheng DJ, Cheng TC, Qin JJ, Duan J, Xu HF, et al: The genome of a lepidopteran model insect, the 
silkworm Bombyx mori. Insect Biochemistry and Molecular Biology 2008, 38:1036-1045

37. Zhang JY, Pan MH, Sun ZY, Huang SJ, Yu ZS, Liu D, Zhao DH, Lu C: The genomic underpinnings of apoptosis in the silkworm, Bombyx mori. BMC Genomics 2010, 11:611.

38. Alnemri ES, Livingston DJ, Nicholson DW, Salvesen G, Thornberry NA, Wong WW, Yuan J: Human ICE/CED-3 Protease Nomenclature. Cell 1996, 87:171.

39. Dorstyn L, Read SH, Quinn LM, Richardson H, Kumar S: DECAY, a Novel Drosophila Caspase Related to Mammalian Caspase-3 and Caspase-7. Journal of Biological Chemistry 1999, 274:30778-30783.

40. Fraser AG, Evan GI: Identification of a Drosophila melanogaster ICE/CED3-related protease, drICE. Embo J 1997, 16:2805-2813.

41. Fraser AG, McCarthy NJ, Evan Gl: drICE is an essential caspase required for apoptotic activity in Drosophila cells. Embo J 1997, 16:6192-6199.

42. Song Z, McCall K, Steller H: DCP-1, a Drosophila Cell Death Protease Essential for Development. Science 1997, 275:536-540.

43. Muro I, Berry DL, Huh JR, Chen CH, Huang H, Yoo SJ, Guo M, Baehrecke EH, Hay BA: The Drosophila caspase Ice is important for many apoptotic cell deaths and for spermatid individualization, a nonapoptotic process. Development 2006, 133:3305-3315

44. Xu D, Wang Y, Willecke R, Chen Z, Ding T, Bergmann A: The effector caspases drICE and dcp-1 have partially overlapping functions in the apoptotic pathway in Drosophila. Cell Death Differ 2006, 13:1697-1706.

45. Bryant B, Ungerer MC, Liu Q, Waterhouse RM, Clem RJ: A caspase-like decoy molecule enhances the activity of a paralogous caspase in the yellow fever mosquito, Aedes aegypti. Insect Biochemistry and Molecular Biology 2010, 40:516-523.

46. Muro I, Monser K, Clem RJ: Mechanism of Dronc activation in Drosophila cells. J Cell Sci 2004, 117:5035-5041

47. Snipas SJ, Drag M, Stennicke HR, Salvesen GS: Activation mechanism and substrate specificity of the Drosophila initiator caspase DRONC. Cell Death Differ 2008, 15:938-945.

48. Chen P, Rodriguez A, Erskine R, Thach T, Abrams JM: Dredd, a Novel Effector of the Apoptosis Activators Reaper, Grim, and Hid in Drosophila. Developmental Biology 1998, 201:202-216.

49. Leulier F, Rodriguez A, Khush RS, Abrams JM, Lemaitre B: The Drosophila caspase Dredd is required to resist gram-negative bacterial infection. EMBO Rep 2000, 1:353-358.

50. Doumanis J, Quinn L, Richardson H, Kumar S: STRICA, a novel Drosophila melanogaster caspase with an unusual serine/threonine-rich prodomain, interacts with DIAP1 and DIAP2. Cell Death Differ 2001, 8:387-394.

51. Harvey NL, Daish T, Mills K, Dorstyn L, Quinn LM, Read SH, Richardson H, Kumar S: Characterization of the Drosophila caspase, DAMM. J Biol Chem 2001, 276:25342-25350

52. Baum JS, Arama E, Steller H, McCall K: The Drosophila caspases Strica and Dronc function redundantly in programmed cell death during oogenesis. Cell Death Differ 2007, 14:1508-1517.

53. Seol DW, Billiar TR: A Caspase-9 Variant Missing the Catalytic Site Is an Endogenous Inhibitor of Apoptosis. Journal of Biological Chemistry 1999, 274:2072-2076.

54. Ng PWP, Porter AG, Jänicke RU: Molecular Cloning and Characterization of Two Novel Pro-apoptotic Isoforms of Caspase-10. Journal of Biological Chemistry 1999, 274:10301-10308.

55. Pauchet $Y$, Freitak D, Heidel-Fischer HM, Heckel DG, Vogel H: Immunity or digestion: glucanase activity in a glucan-binding protein family from Lepidoptera. J Biol Chem 2009, 284:2214-2224.

56. d'Alencon E, Sezutsu H, Legeai F, Permal E, Bernard-Samain S, Gimenez S, Gagneur C, Cousserans F, Shimomura M, Brun-Barale A, et al: Extensive synteny conservation of holocentric chromosomes in Lepidoptera despite high rates of local genome rearrangements. Proc Natl Acad Sci USA 2010, 107:7680-7685.

57. Nei $M$, Gojobori T: Simple methods for estimating the numbers of synonymous and nonsynonymous nucleotide substitutions. Mol Biol Evol 1986, 3:418-426

58. Nei M: Molecular evolution and phylogenetics. New York: Oxford Univ. Press; 2000.

59. Gu X: Statistical methods for testing functional divergence after gene duplication. Molecular Biology and Evolution 1999, 16:1664-1674.

60. Conant GC, Wagner A: Asymmetric Sequence Divergence of Duplicate Genes. Genome Research 2003, 13:2052-2058.
61. Panchin A, Gelfand M, Ramensky V, Artamonova I: Asymmetric and nonuniform evolution of recently duplicated human genes. Biology Direct 2010, 5:54.

62. Lynch M, Conery JS: The Evolutionary Fate and Consequences of Duplicate Genes. Science 2000, 290:1151-1155.

63. Hughes AL: The Evolution of Functionally Novel Proteins after Gene Duplication. Proceedings of the Royal Society of London Series B: Biological Sciences 1994, 256:119-124.

64. Pauchet $Y$, Wilkinson $P$, Vogel H, Nelson DR, Reynolds SE, Heckel DG, ffrench-Constant RH: Pyrosequencing the Manduca sexta larval midgut transcriptome: messages for digestion, detoxification and defence. Insect Mol Biol 2010, 19:61-75.

65. Freitak $D$, Wheat $C W$, Heckel DG, Vogel H: Immune system responses and fitness costs associated with consumption of bacteria in larvae of Trichoplusia ni. BMC Biol 2007, 5:56.

66. Fischer HM, Wheat CW, Heckel DG, Vogel H: Evolutionary origins of a novel host plant detoxification gene in butterflies. Mol Biol Evol 2008, 25:809-820.

67. Thompson JD, Gibson TJ, Plewniak F, Jeanmougin F, Higgins DG: The CLUSTAL_X windows interface: flexible strategies for multiple sequence alignment aided by quality analysis tools. Nucl Acids Res 1997 25:4876-4882.

68. Insecta Central. [http://insectacentral.org].

69. Papanicolaou A, Stierli R, ffrench-Constant RH, Heckel DG: Next generation transcriptomes for next generation genomes using est2assembly. BMC Bioinformatics 2009, 10:447.

70. McIntosh AH, Ignoffo CM, Quhou C, Pappas M: Establishment of a cell ine from Heliothis armigera (Hbn.) (Lepidoptera: Noctuidae). In Vitro 1983, 19:589-590.

71. Katoh K, Toh H: Recent developments in the MAFFT multiple sequence alignment program. Brief Bioinform 2008, 9:286-298.

72. Ronquist F, Huelsenbeck JP: MrBayes 3: Bayesian phylogenetic inference under mixed models. Bioinformatics 2003, 19:1572-1574.

73. Tamura K, Dudley J, Nei M, Kumar S: MEGA4: Molecular Evolutionary Genetics Analysis (MEGA) software version 4.0. Mol Biol Evol 2007, 24:1596-1599.

74. LaCount DJ, Hanson SF, Schneider CL, Friesen PD: Caspase inhibitor P35 and inhibitor of apoptosis Op-IAP block in vivo proteolytic activation of an effector caspase at different steps. J Biol Chem 2000, 275:15657-15664.

75. GU X, Vander Velden K: DIVERGE: phylogeny-based analysis for functionalstructural divergence of a protein family. Bioinformatics 2002, 18:500-501.

76. ExPASy ScanProsite. [http://www.expasy.ch/tools/scanprosite]

77. Kaikobase. [http://sgp.dna.affrc.go.jp/KAlKObase/].

78. Shimomura M, Minami H, Suetsugu Y, Ohyanagi H, Satoh C, Antonio B, Nagamura $Y$, Kadono-Okuda K, Kajiwara H, Sezutsu H, et al: KAIKObase: an integrated silkworm genome database and data mining tool. BMC Genomics 2009, 10:486.

doi:10.1186/1471-2164-12-357

Cite this article as: Courtiade et al: A comprehensive characterization of the caspase gene family in insects from the order Lepidoptera. $B M C$ Genomics 2011 12:357.

\section{Submit your next manuscript to BioMed Central and take full advantage of:}

- Convenient online submission

- Thorough peer review

- No space constraints or color figure charges

- Immediate publication on acceptance

- Inclusion in PubMed, CAS, Scopus and Google Scholar

- Research which is freely available for redistribution 